Black-hole formation from stellar collapse

This content has been downloaded from IOPscience. Please scroll down to see the full text. 2003 Class. Quantum Grav. 20 S73

(http://iopscience.iop.org/0264-9381/20/10/309)

View the table of contents for this issue, or go to the journal homepage for more

Download details:

IP Address: 141.211.4.224

This content was downloaded on 05/09/2015 at 08:40

Please note that terms and conditions apply. 


\title{
Black-hole formation from stellar collapse
}

\author{
Chris L Fryer \\ MS B210, Theoretical Astrophysics, Los Alamos National Laboratory, Los Alamos, \\ NM 87545, USA \\ E-mail: fryer@lanl.gov
}

Received 2 October 2002

Published 25 April 2003

Online at stacks.iop.org/CQG/20/S73

\begin{abstract}
I review the end-state of massive stellar evolution, following the evolution of these massive stars from the onset of collapse through the formation of a compact remnant and the possible supernova or hypernova explosion. In particular, I concentrate on the formation of black holes from stellar collapse: the fraction of stars that form black holes, the black-hole mass distribution and the velocities these black-hole remnants may receive during their formation process.
\end{abstract}

PACS number: 97.60.Bw

\section{Supernovae, hypernovae and black-hole formation}

Stellar-massed black holes are observed in the universe through the accretion of matter. Most of these observations arise when a binary companion loses mass which accretes onto the black hole (e.g., x-ray binaries). Interpretations of the observations of these binaries have yielded black-hole mass distributions ranging from delta function distributions (Bailyn et al 1998) to a range of black-hole masses which depend upon the orbital period of the pre-collapse system (Lee and Brown 2002). Unfortunately, uncertainties in and the scarcity of the observations make it very difficult to determine the true mass distribution of black holes. However, this picture will change with gravitational wave observations of compact binaries, which may increase the number of black-hole systems by over an order of magnitude.

Stellar-mass black holes form during the final stages of a massive star's life, when the star implodes on itself. To understand black-hole formation, we must also understand which stars collapse and the explosions that are produced by stellar collapse. Type Ib/c and Type II supernovae (Filippenko 1997), most hypernovae ${ }^{1}$ (Nakamura et al 2001) and possibly longduration gamma-ray bursts (Woosley 1993, MacFadyen and Woosley 1993) are all believed to be produced by the gravitational energy released when the core of a massive $\left(\sim 8-10 \mathrm{M}_{\odot}\right.$

1 We restrict the definition of hypernova in this paper to those jet-driven explosions (see Heger et al 2002) that are not associated with gamma-ray bursts. 
or greater) star collapses down to a neutron star or black hole. Less massive stars eject most of their material prior to core-collapse and instead form white dwarfs. Below we discuss the relation between explosion and remnant type: supernovae versus gamma-ray bursts, neutron star (NS) versus black-hole (BH) remnants. These different outcomes depend not only on the star's mass, but also on its metallicity (which effects its mass loss through winds), its rotation rate and its binary companion.

\subsection{Collapse versus envelope ejection}

Stars are powered by the fusion of elements in their core. Hydrogen burning into helium fuels most of a star's life. When the hydrogen fuel is used up, massive stars will contract, heat up and ignite the ashes of hydrogen burning (helium), accessing a new store of fuel. For massive stars, this process of burning the ashes of each preceding burning stage can continue through carbon, oxygen and even silicon burning until an iron core is built up in the centre of the star. Because iron is at a minimum in energy states, the fusion of iron does not produce further energy and burning halts (regardless of a star's mass). The iron core is supported by electron degeneracy and thermal pressures, but when the core reaches too large a mass, the high temperatures in the core cause the iron to dissociate into alpha particles, an endothermic reaction which reduces the thermal pressure. In addition, as the core reaches higher and higher densities, electrons begin to capture onto protons, removing electron degeneracy pressure. This loss in pressure causes the iron core to contract which accelerates the rate at which iron dissociation and electron capture occurs. Very quickly the core-collapse becomes a runaway process.

For lower mass stars, the contracting core does not heat up sufficiently to produce an iron core before shell burning above the core ejects the star's envelope, ultimately leaving behind a white dwarf. The transition from white dwarf (at lower masses) and core-collapse (at higher masses) depends sensitively on the shell burning phases and probably occurs somewhere between $8-10 \mathrm{M}_{\odot}$.

\subsection{Neutron star versus black hole}

For stars more massive than $8-10 \mathrm{M}_{\odot}$, a runaway collapse proceeds until the stellar core reaches nuclear densities and neutron degeneracy pressure and nuclear forces halt the collapse. The 'bounce' of the core launches a shock back through the star. Although this bounce was once believed to drive the explosion, it was found that neutrino emission from the shock causes it to stall. Plenty of energy to drive an explosion is left in the hot proto-neutron star and neutrinos emitted from this core can heat the stalled region and revive the shock. The stalled shock region, which stretches from the $\sim 30 \mathrm{~km}$ collapsed core (also known as proto-neutron star) out to the furthermost edge of the shock $(\sim 100-300 \mathrm{~km})$ is convectively unstable (figure 1). Neutrinos from the hot proto-neutron star heat the bottom of the convective region, further driving this convection which converts the thermal energy from neutrino deposition into kinetic energy. If the convective region can overcome the ram pressure of the outer layers of the star which are crashing down on it, an explosion is launched.

Strong explosions eject most of the star, ultimately leaving behind a neutron star which we observe as pulsars or, through accretion, as x-ray binaries. If only a weak explosion is launched, some of the ejecta may fall back and accrete onto the neutron star. In some cases, this material can be sufficient to cause the neutron star to collapse and form a black hole. In still other cases, the convective region is never able to overcome the ram pressure of the shock, and the shock eventually moves in, compressing the proto-neutron star until it collapses to form a black hole. 


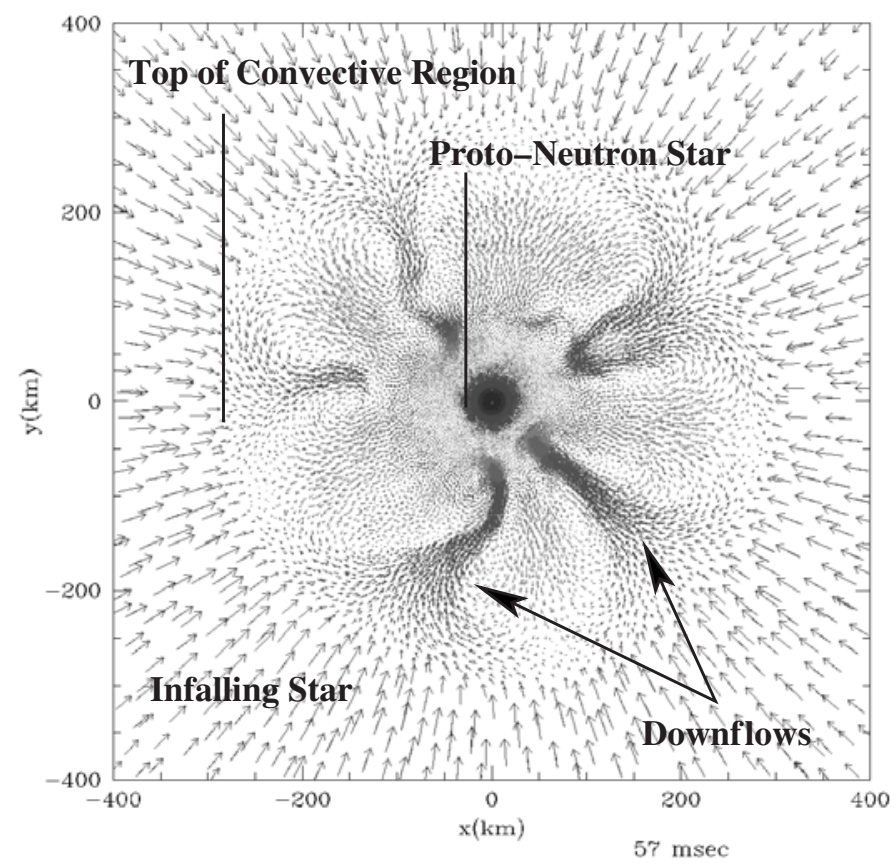

Figure 1. Slice of a three-dimensional simulation from Fryer and Warren (2002). The infalling material crashes down on the top of the convection region and the ram pressure from this material prevents an explosion. This material builds up and accretes in discrete downflows onto the protoneutron star. Neutrinos from the proto-neutron star and this accretion heat the material in the convective region causing it to rise. When this material can overcome the lid produced by the infalling material, this pressure cooker explodes!

Table 1. Collapse of a $60 \mathrm{M}_{\odot}$ star-effects of winds (Fryer et al 2002).

\begin{tabular}{lclc}
\hline Wind multiplier & Final mass & Explosion energy & Remnant mass \\
\hline 1 & $3.132 \mathrm{M}_{\odot}$ & $1.45 \times 10^{51} \mathrm{erg}$ & $1.35 \mathrm{M}_{\odot}$ \\
$1 / 2$ & $4.389 \mathrm{M}_{\odot}$ & $2.36 \times 10^{51} \mathrm{erg}$ & $1.17 \mathrm{M}_{\odot}$ \\
$1 / 3$ & $6.108 \mathrm{M}_{\odot}$ & $1.60 \times 10^{51} \mathrm{erg}$ & $2.11 \mathrm{M}_{\odot}$ \\
$1 / 4$ & $7.550 \mathrm{M}_{\odot}$ & $0.30 \times 10^{51} \mathrm{erg}$ & $5.2 \mathrm{M}_{\odot}$ \\
$1 / 6$ & $10.746 \mathrm{M}_{\odot}$ & $0 \mathrm{erg}$ & $10.7 \mathrm{M}_{\odot}$ \\
\hline
\end{tabular}

Ignoring the effects of binary companions, stellar winds and rotation, the fate of a massive star depends solely upon the mass of the star. Fryer (1999b) found that as a star's mass increases, its fate evolves from a strong explosion with a neutron star remnant through a weak explosion with a black-hole remnant to no explosion whatsoever with a black-hole remnant (figure 2). The masses where these transitions occur are at roughly $20 \mathrm{M}_{\odot}$ and $\sim 45 \mathrm{M}_{\odot}$, respectively.

But the effects of rotation, winds and binaries cannot be neglected. Mass loss from stellar winds can have a dramatic effect on the fate of massive stars (table 1). A factor of 2 decrease in the mass-loss rate can change the collapse remnant from a $2.0 \mathrm{M}_{\odot}$ neutron star to a $>10 \mathrm{M}_{\odot}$ black hole and can change a strong supernova explosion to no explosion whatsoever. Current models for massive stellar winds assume steady mass loss and depend upon parameters that can only be determined through observations, not basic physics. Unfortunately, observations of massive, mass-losing stars have shown that mass loss is clumpy, sporadic and difficult to 


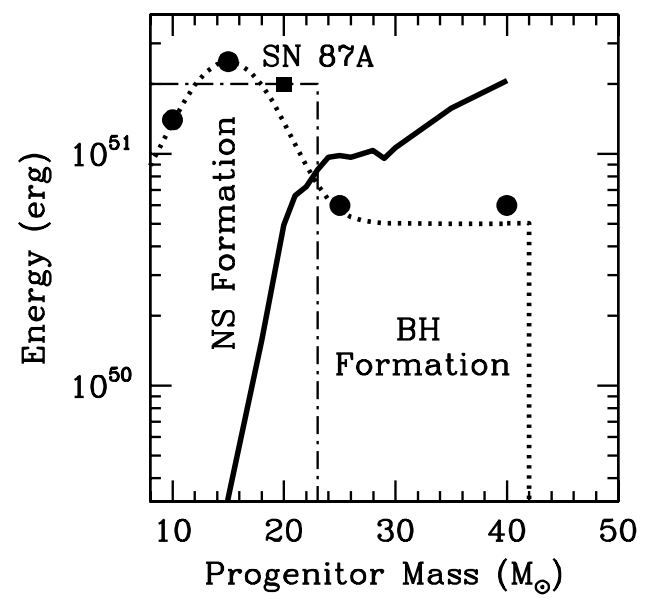

Figure 2. Binding energy (solid line)/explosion energy (dotted, dot-dashed) line versus progenitor star mass for single star models with no mass loss. The dotted line is the fit to explosion models (circles) by Fryer (1999a). The dot-dashed line refers to a step-function explosion energy used to try to fit the mass-gap in black-hole masses. The square represents SN 1987A for which we truly know the progenitor mass.

predict and it is not clear that mass-loss rates used in stellar models are accurate to better than an order of magnitude.

Many have argued that a binary companion can spin up a massive star but the magnitude of the spin up is still a matter of debate (e.g., Paczynski 1998, Fryer et al 1999, Brown et al 2000). The undisputed effect of binaries is to help eject mass through mass transfer or common envelope episodes. When a massive star expands out of hydrogen burning onto the giant branch, it can lose mass to its companion (or even out of its entire binary system) through interactions with its companion. This can cause the star to enter a Wolf-Rayet (rapid mass losing) phase earlier and make wind mass loss more effective. Unfortunately, roughly half of all the stars in our Galaxy are in sufficiently close binaries that these binary effects must be included.

Simulations by Fryer and Heger (2000) showed that rotating stars have weaker explosions via the neutrino driven mechanism than non-rotating stars. Note, however, that rotating stars may ultimately yield gamma-ray bursts or hypernovae and drive strong explosions. This occurs because the angular momentum profiles produced in the current stellar models (Heger 1998) prevent convection and the energy transport that convection affords. The collapse simulations by Fryer and Heger (2000) preserved angular momentum exactly. If convection in the core leads to angular momentum transport, such as in the latest three-dimensional models by Fryer and Warren (2002), the convection is not damped as much (figure 3) and may lead to explosions on a par with non-rotating counterparts. However, the angular momentum transport for the simulation in figure 3 is likely to be higher than what actually occurs in the core, and it may be that the Fryer and Heger (2000) results are closer to nature than what is shown in figure 3 . Unfortunately, although there now exist progenitors for rotating massive stars at collapse, these simulations are missing several pieces of crucial physics (most notable being magnetic fields) and it is not clear how fast massive stellar cores are truly rotating.

Despite these uncertainties (as well as uncertainties in the initial mass function), Fryer and Kalogera (2001) showed that between 5-40\% of all the collapsing stars form black holes. That means there are roughly $5 \times 10^{6}-4 \times 10^{7}$ black holes in our Galaxy. 

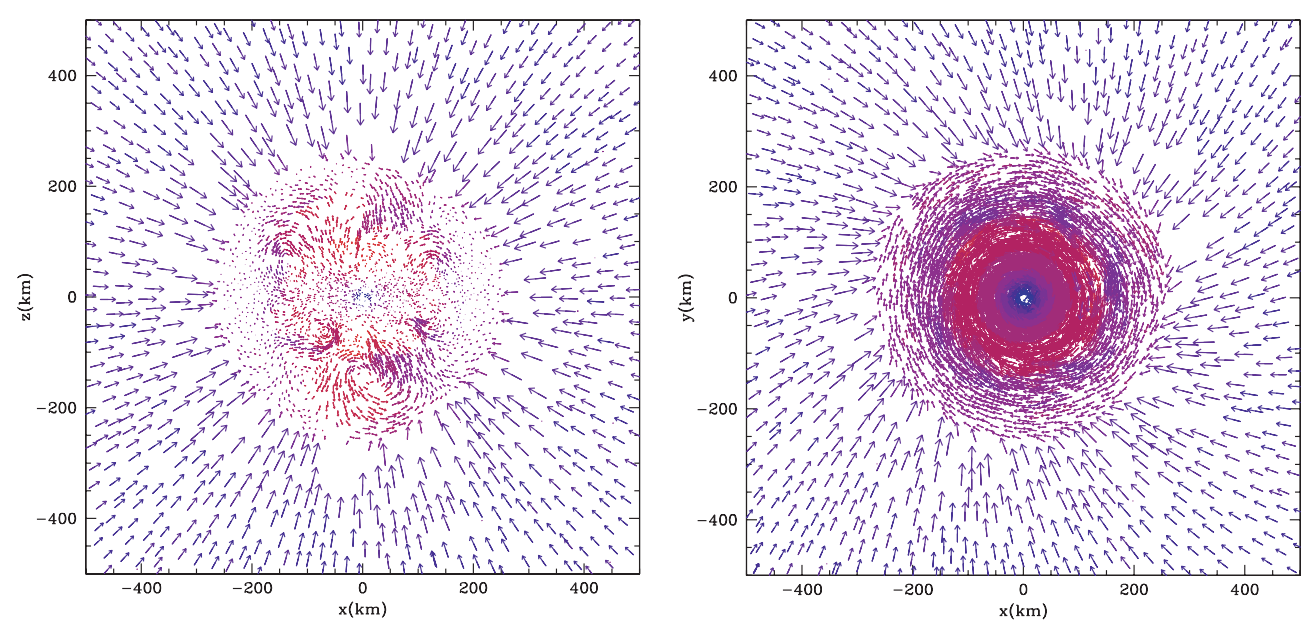

Figure 3. Slices of a three-dimensional simulation of a rotating $15 \mathrm{M}_{\odot}$ star (Fryer and Warren 2003) looking along the equatorial (left) and polar (right) axes. Angular momentum changes the convection dramatically, but not as much as predicted by Fryer and Heger (2000) where the angular momentum is not transported whatsoever!

(This figure is in colour only in the electronic version)

\section{Black-hole mass distribution}

The gravitational wave emission from black-hole inspiral (either into a large black hole producing a LISA signal or into smaller black holes producing a strong LIGO signal) depends sensitively on the black-hole mass (Sipior and Sigurdsson 2002). With the caveats of the uncertainties listed above, it is possible to also derive a black-hole mass distribution from theory. From BH x-ray binary systems, we can also derive a black-hole mass distribution, and depending upon how you analyse these results, the observational distribution may or may not be consistent with the theoretical distribution. Here we summarize the theoretical predictions for black-hole mass distributions, how they constrain stellar models and how they compare with observational data.

\subsection{Theoretical mass distributions}

Fryer and Kalogera (2001) did a systematic study of the remnants produced by stellar collapse using the explosion energies from figure 2 as a guide. The binding energy of a star increases dramatically with mass between 15 and $25 \mathrm{M}_{\odot}$ and it is likely that the explosion energy decreases in this same range, so even with the uncertainties in the supernova explosion mechanism, the transition mass from NS and $\mathrm{BH}$ remnants is defined within a narrow mass range. In the absence of stellar winds, the uncertainty in the binding explosion energies as a function of progenitor mass does not make large uncertainties in the black-hole mass distribution. Using the fit to the supernova explosion energy and binding energies of non-mass losing stars (figure 2), Fryer and Kalogera constructed a mass distribution of stellar-mass black holes (figure 4). The slope of this distribution is fairly insensitive to the fit to the explosion energy.

However, if one includes the effects of mass loss, especially mass loss enhanced by binary interactions, this picture can change quite dramatically. In fact, recent models of massive stars (Woosley et al 1993) predicted that massive stars above 30-40 $\mathrm{M}_{\odot}$ would lose most of their mass through winds. With such strong winds, if these stars lose their hydrogen envelopes 


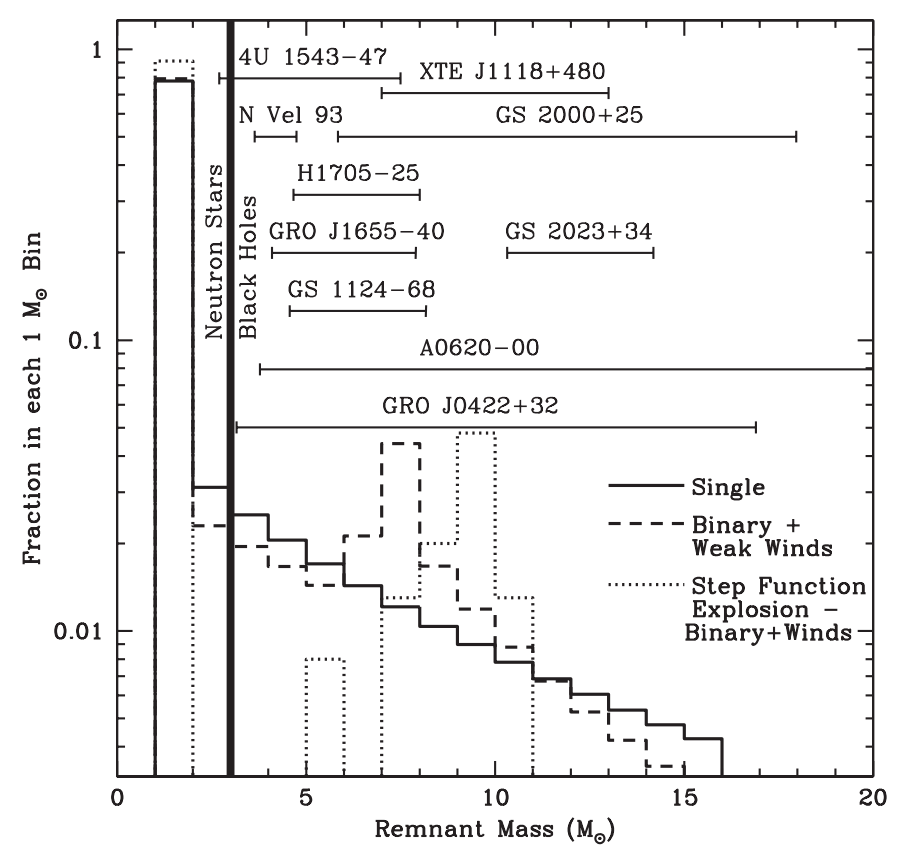

Figure 4. Mass distribution of black holes and neutron stars using the best fit to the Fryer (1999a) explosion energies for single stars (solid line) and binary stars with weak winds (dashed line) along with the step-function explosion energy for binary stars and weak winds (dotted lines). The data of observed systems with their error bars are plotted as well.

prior to helium ignition, it is likely that they will end their lives with masses less than 4-5 $\mathrm{M}_{\odot}$. Such high mass-loss rates would make it difficult to form x-ray binaries with masses above 7-8 $\mathrm{M}_{\odot}$. The issue of mass loss has been a point of contention between the x-ray binary community and the stellar community for over a decade. Although the stellar community is working on the theory of mass loss and clearly understands the physics of stellar winds better than the x-ray binary community, the x-ray binary community has the upper hand in the fact that close binaries with $10 \mathrm{M}_{\odot}$ black holes exist in the Galactic disk (the progenitor probably had a metallicity near solar). Stellar evolutionists have been forced to slowly decrease the mass-loss rate of stars. A factor of 6 decrease in mass-loss factors (table 1) easily allows more massive BHs and mass distributions close to the binary mass distribution derived by Fryer and Kalogera (figure 4).

These two mass distributions predict a continuous distribution of black-hole masses from the maximum neutron star mass up to $\sim 10 \mathrm{M}_{\odot}$. However, many black-hole binary observers argue strongly that there is a gap in black-hole masses from 3-5 $\mathrm{M}_{\odot}$. How must the theory change to incorporate this? Fryer and Kalogera chose a step function for the supernova explosion energy (figure 2) and found that they could produce a black-hole mass distribution with a gap (figure 4). However, as we shall discuss below, the evidence for the mass gap is marginal at best, and supernova observations suggest strongly that a step function in the explosion energy is not possible.

The basic trends in the various uncertainties are summarized in table 2 . Winds are the biggest effect and can change the total number of $\mathrm{BHs}$ by over an order of magnitude. These effects are enhanced in close binaries. Uncertainties in the initial mass function (IMF) are also important (factors of 2 in the total black-hole mass number). Uncertainties in the explosion energy make the smallest differences but can change results by $50 \%$. 
Table 2. Trends in black-hole masses.

\begin{tabular}{llll}
\hline Physical property & Effect & Maximum BH mass & Number of BH \\
\hline Increasing rotation & Decrease energy & No change & Increase \\
Increasing wind & More mass loss & Decrease & Decrease \\
Close binary & Increase wind & Decrease & Decrease \\
Steep IMF & Fewer massive stars & No change & Decrease \\
\hline
\end{tabular}

If the star is rotating fast enough, a disk can form around the black hole. The accretion of this disk can lead to gamma-ray bursts or hypernovae which prevents further accretion onto the black hole, producing even more low-mass black holes than Fryer and Kalogera (2001) predicted. Observations of hypernovae (Nomoto et al 2002) show that most stars above $20 \mathrm{M}_{\odot}$ produce hypernovae or gamma-ray bursts. However, some weak explosions have been observed with likely progenitors above $20 \mathrm{M}_{\odot}$. Due to their low nickel yield (a factor of 100 less than hypernovae) and hence, low luminosity, there are strong biases against observing these weak supernovae. Even though hypernovae dominate the observations, it is likely that weak or no explosions are the more common fate of massive stars, and the Fryer and Kalogera (2001) distribution is probably close to the correct black-hole distribution.

\subsection{Comparing with observations}

Theory clearly does not predict a delta function mass distribution. Creating a delta function mass distribution at $10 \mathrm{M}_{\odot}$ for close binary systems is difficult, if not impossible to do. It would require a step function in the explosion energy which is all but ruled out by the observed weak supernovae such as SN 1997D (Turatto et al 1998). It also would require that the progenitor of these close binaries not lose its entire hydrogen envelope during a mass transfer phase prior to collapse. Figure 4 shows the observed black-hole masses with their associated error bars. Fryer and Kalogera (2001) argued that these observations are actually more consistent with a continuous mass distribution like that we would expect from theory. If hypernovae account for the formation of many black holes, theory produces even more black holes where observers claim there is a gap.

Given that these effects are unlikely, if not impossible, it is worth discussing some of the biases that might effect the observational sample. Fryer and Kalogera (2001) did not see this gap in the data and they argued that the gap only manifests itself when some of the observations are discarded. These data are generally discarded on grounds that they 'aren't reliable', but I fear that they are discarded because the error bar on the mass dips below $3 \mathrm{M}_{\odot}$ and hence the compact object could be a NS. But by discarding those BHs with masses which could be below $3 \mathrm{M}_{\odot}$, observers are biasing against low-mass BHs.

Of course, there can be other physical effects to cause the observational data set to have a gap in the 3-5 $\mathrm{M}_{\odot}$. First of all, low-mass BHs are formed in supernova explosions that eject considerable mass which make it more difficult for the binary to remain bound. In addition, depending upon the kick mechanism for NSs, low-mass BHs might receive stronger kicks than high-mass BHs, unbinding these binaries.

\subsection{Black-hole kicks}

It is now generally accepted that not only are NSs born with fast velocities as high as $1000 \mathrm{~km} \mathrm{~s}^{-1}$ (Cordes et al 1993), but their 'kick' distribution appears to be bimodal with roughly half of all NSs receiving velocities above $400-500 \mathrm{~km} \mathrm{~s}^{-1}$ and the other half receiving 
only 100-500 $\mathrm{km} \mathrm{s}^{-1}$ kicks (Cordes and Chernoff 1998). Given that BHs are formed in similar stellar collapses to those that form NSs, it is not surprising that black holes may also receive 'kicks' at birth.

Unfortunately, unlike NSs which can emit quite brightly as single objects (pulsars), BHs are currently observed only in binary systems where accretion onto the black hole powers $\mathrm{x}$-ray emission. So it is much harder to get a population of BHs from which to derive a kick distribution. Those black holes that are observed are in binaries. Not only must we model the entire binary to extract a kick velocity, but we must also be aware of the biases that are involved in limiting our sample to those black holes that actually remain bound.

Fryer (1999a) studied the currently (at that time) known BH binary systems and found that although all but one binary system needed a kick to explain its peculiar velocities, for all systems, the BH could have received a kick as high as $50 \mathrm{~km} \mathrm{~s}^{-1}$ at birth and a Maxwellian kick distribution with an rms velocity of $50 \mathrm{~km} \mathrm{~s}^{-1}$ fits all of the binary systems much better than a 0 velocity kick distribution. Theories of kick formation are not developed enough to really say much about the neutron star kick distribution, let alone the black-hole kick distribution. But assuming that kicks are produced in all stars that produce supernovae and since most black holes are produced in supernovae which have extensive fallback, it would not be surprising that the momentum of the kick imparted to NSs is the same as BHs, implying that the velocity of black-hole kicks is $v_{\mathrm{BH}}=v_{\mathrm{NS}} M_{\mathrm{NS}} / M_{\mathrm{BH}}$ (Fryer 1999a).

This completes our review of the current understanding of the formation of stellar-massed BHs, their mass distribution and their velocities.

\section{References}

Bailyn C D, Jain R K, Coppi P and Orosz J A 1998 Astrophys. J. 499367

Brown G E, Lee C-H, Wijers R A M J, Lee H K, Israelian G and Bethe H A 2000 New Astron. 5191

Cordes J M and Chernoff D F 1998 Astrophys. J. 505315

Cordes J M, Romani R W and Lundgren S C 1993 Nature 362133

Filippenko A V 1997 Ann. Rev. Astron. Astrophys. 35309

Fryer C L 1999a Proc. IAU Symposium 195 vol 3 (San Francisco, CA: ASP) p 339

Fryer C L 1999b Astrophys. J. 522413

Fryer C L and Heger A 2000 Astrophys. J. 5411033

Fryer C L, Heger A, Langer N and Wellstein S 2002 Astrophys. J. 578335

Fryer C L and Kalogera V 2001 Astrophys. J. 554548

Fryer C L and Warren M S 2002 Astrophys. J. 574 L65

Fryer C L and Warren M S 2003 Astrophys. J. in preparation

Fryer C L, Woosley S E and Hartmann D H 1999 Astrophys. J. 526152

Heger A, Fryer C L, Woosley S E, Langer N and Hartmann D 2002 Preprint astro-ph/0212469 (Astrophys. J. at press) Lee C-H and Brown G E 2002 Preprint astro-ph/0208304

MacFadyen A I and Woosley S E 1993 Astrophys. J. 550410

Nakamura T, Umeda H, Iwamoto K, Nomoto K, Hashimoto M, Hix W R and Thielemann F-K 2001 Astrophys. J. $\mathbf{5 5 5} 880$

Nomoto K, Maeda K, Umeda H, Ohkubo T, Deng J and Mazzali P 2002 A Massive Star Odyssey, from Main Sequence to Supernova (Proc. IAU Symposium 212) ed K A van der Hucht, A Herrero and C Esteban (San Francisco, CA: ASP) at press

Orosz J A Private communication

Paczynski B 1998 Astrophys. J. 494 L45

Sipior M S and Sigurdsson S 2002 Astrophys. J. 572962

Turatto M et al 1998 Astrophys. J. 498 L129

Woosley S E 1993 Astrophys. J. 405273

Woosley S E, Langer N and Weaver T A 1993 Astrophys. J. 411823 